\title{
Combination Therapy to Treat Asian Female Pattern Hair Loss
}

\author{
Yohei Tanaka1,2*(), Toru Aso², Jumpei Ono $^{2}$ \\ ${ }^{1}$ Clinica Tanaka Plastic, Reconstructive Surgery and Anti-Aging Center, Matsumoto, Nagano, Japan \\ ${ }^{2}$ AGA Skin Clinic, Tokyo, Japan \\ Email: ^info@clinicatanaka.jp
}

How to cite this paper: Tanaka, Y., Aso, T. and Ono, J. (2019) Combination Therapy to Treat Asian Female Pattern Hair Loss. Journal of Cosmetics, Dermatological Sciences and Applications, 9, 216-222.

https://doi.org/10.4236/jcdsa.2019.93018

Received: August 5, 2019

Accepted: August 30, 2019

Published: September 3, 2019

Copyright $\odot 2019$ by author(s) and Scientific Research Publishing Inc. This work is licensed under the Creative Commons Attribution International License (CC BY 4.0).

http://creativecommons.org/licenses/by/4.0/

\begin{abstract}
Background: Hair loss in women has a various psychosocial impact and effective treatments have been required. Camouflaging products which cover exposed areas on the scalp and hide visible hair loss are common, but medical therapies for female pattern hair loss (FPHL) are not so common. We have treated patients with FPHL using our combination therapy that consists of oral spironolactone, oral and topical minoxidil, and an injectable treatment for six years. Methods: 4568 female patients were treated between the years 2013 and 2018. Our combination therapy consists of $25 \mathrm{mg}$ oral spironolactone once daily, $2.5 \mathrm{mg}$ oral and $5 \%$ solution topical minoxidil twice daily, and an injectable treatment once monthly for 6 - 12 months. Digital photographs were taken pre- and post-treatment, and patient assessments were recorded after 6 and 12 months post-treatment. Results: Significant improvement was observed compared to the pre-treatment for all patients in the digital photographs. $95 \%$ and $96 \%$ of patients reported satisfaction with the results of the treatment after 6 and 12 months post-treatment, respectively. Minor complications were observed in a total of 223 (4.9\%) patients, characterized by slight pain and bleeding due to injection, slight palpitation, swelling, hair growth in undesirable areas, headache, dizziness, itching. The seminor complications resolved spontaneously. No treatment-related adverse events were observed. Conclusion: A combination of these therapeutic options offers rapid and highly efficacious treatment for FPHL with minimal complications, and is recommended as an effective treatment for FPHL.
\end{abstract}

\section{Keywords}

Combination Therapy, Female Patternhair Loss (FPHL), Minoxidil, Spironolactone 


\section{Introduction}

Female pattern hair loss (FPHL) is characterized by a reduction in the number of terminal fibers per follicular unit over the crown and frontal scalp with relative sparing of the anterior hair line [1]. The age of onset of FPHL is later than that seen in men. Twelve percent of women develop detectable FPHL by age 29 years, $25 \%$ by age 49 years, $41 \%$ by 69 years, and over $50 \%$ have some element of FPHL by 79 years. Only $43 \%$ of women aged 80 years and above show no evidence of FPHL [2]. Hair loss in women has a various psychosocial impact on the affected individual, and especially causes emotional distress. Women place a greater emphasis than men on physical appearances and outward attractiveness [1] [2]. Women have been more likely than men to have a lowered quality of life [3], and to restrict social contacts as a result of hair loss [4].

While the role of androgens in the pathogenesis of male pattern hair loss (MPHL) has been clearly established, the role of androgens in FPHL is less clear [1]. Lifestyle choices, including diet, sleep patterns, and psychological pressure might influence the progression of FPHL. The pattern of hair loss seen in women is materially different to men. Bitemporal recession is less pronounced and vertex bald spots are almost never seen [1].

Various medical therapies have been used to treat FPHL, including topical minoxidil, topical $17 \alpha$-estradiol, oral anti-androgen agents, mineral supplements, growth factors, surgery, and light treatment have been used [5] [6]. Combination therapies are often preferred, because combining medications with different mechanisms of action enhances the efficacy [5] [6] [7].

We have experienced clinically satisfactory results achieved by a combination therapy including oral, topical and injectable treatments [7]. In this study, we have used oral spironolactone, oral and topical minoxidil, and injectable treatments. Spironolactone acts as an androgen antagonist by blocking androgen receptors, as well as inhibiting ovarian androgen production [8] [9]. Minoxidil induces cutaneous blood flow [5] [6] [10] [11] [12].

We evaluated the efficacy of our combination therapy for FPHL in the study. We sought to quantitatively demonstrate the results of our combination therapy for FPHL by retrospectively reviewing and describing our cumulative experience over the past six years.

\section{Methods}

\subsection{Japanese Patients}

In this study, 4568 female patients aged 19 to 83 years (mean age, $39.9 \pm 11.1$ years) were enrolled. Patients underwent our FPHL combination therapy between 2013 and 2018. All of the patients had visited the Tokyo Beauty Clinic to treat FPHL. Since oral minoxidil could decrease blood pressure, patients under oral treatment for hypertension were excluded from this study as well. None of the patients had a history of any type of skin disease or other hair restoration procedure. As this study involved a retrospective review of patients previously 
treated, the approval of an ethics committee was not required. All patients were informed about the risks, benefits and alternative treatments and all provided an informed consent based on the 1975 Declaration of Helsinki. All patients in figures agreed to publication of results and images.

\subsection{Combination Therapy}

Our combination therapy consists of $25 \mathrm{mg}$ oral spironolactone once daily, 2.5 mg oral and 5\% solution topical minoxidil twice daily, and an injectable treatment once monthly for more than 6 months. The injectable treatment $(4 \mathrm{~mL}$ per treatment) is composed of $2 \mathrm{~mL}$ of $0.5 \%$ lidocaine, $0.3 \mathrm{~mL}$ of $2 \%$ minoxidil, and $1.7 \mathrm{~mL}$ of hair regrowth therapy injection (Mesoline hair, MD Skin Solutions, Luxembourg). Mesoline haircontains arginine, aspartic acid, caffeine, copper tripeptide, lysine, niacin, panthenol, propanediol, propylen glycol, retinyl palmitate, pyridoxine, sodium hyaluronate, and ubiquinone-all of which was injected directly into areas where more hair was desired [7]. Postoperative follow-up was scheduled after six and 12 months.

\subsection{Subjective Patients' Assessments}

Subjective assessments were performed using questionnaire data collected six and 12 months post-treatment. Patients rated their degree of satisfaction with improvement of the treated area. Scores were based on a 5-point scale ranging from 0 to 4 ( 0 = worse; 1 = little satisfaction or not satisfied; 2 = fairly satisfied; 3 $=$ satisfied; and $4=$ very satisfied).

\section{Results}

Significant improvement was observed compared to the pre-treatment for all patients in digital photographs (Figure 1 and Figure 2). $95 \%$ and $96 \%$ of patients reported satisfaction with the results of the treatment after 6 and 12 months post-treatment, respectively (Figure 3).
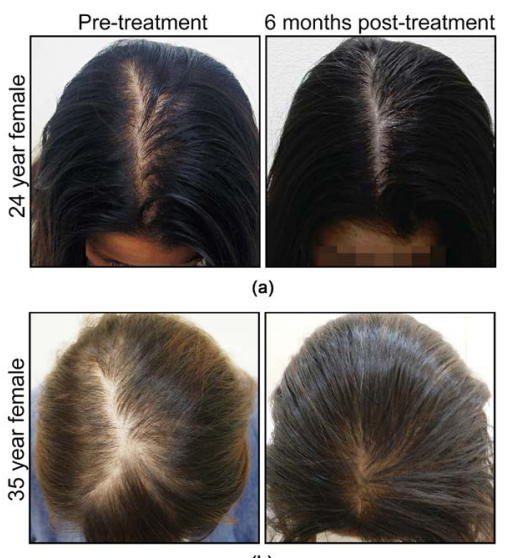

(b)

Figure 1. Representative photographs of hair re-growth, before and after treatment. (a) 24-year-old female pretreatment and 6 months after treatment; (b) 35-year-old female pretreatment and 6 months after treatment. 

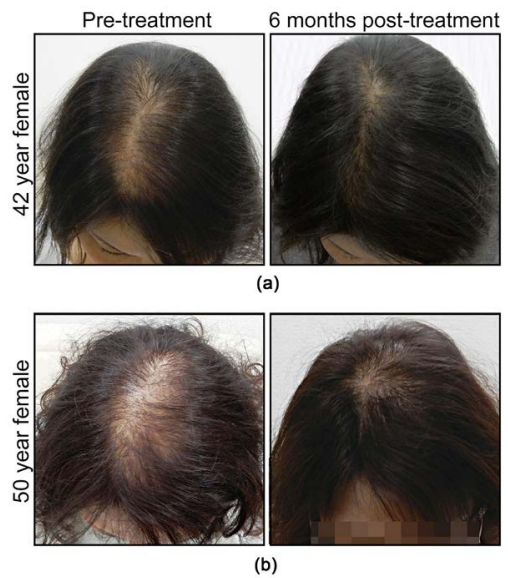

Figure 2. Representative photographs of hair re-growth, before and after treatment. (a) 42-year-old female pretreatment and 6 months after treatment; (b) 50-year-old female pretreatment and 6 months after treatment.

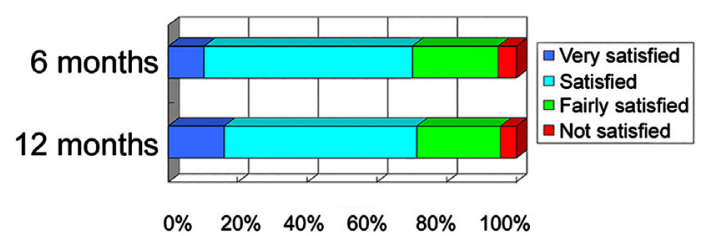

Figure 3. Subjective satisfaction with treatment results after 6 and 12 months post-treatment.

Minor complications were observed in a total of 223 (4.9\%) patients out of 4568 patients. Slight pain due to injection was found in 121 patients (2.6\%), and slight bleeding was found in 115 patients (2.5\%). Slight palpitation, swelling, hair growth in undesirable areas such as face and extremities, headache, dizziness, anditching were found in 119 patients (2.6\%), 64 patients (1.4\%), 58 patients (1.3\%), 55 patients $(1.2 \%), 50$ patients $(1.1 \%)$, and 20 patients $(0.44 \%)$, respectively. Menstrual irregularities were observed in 9 patients $(0.2 \%)$. The seminor complications resolved spontaneously despite continued treatment. No treatment-related adverse events were observed.

\section{Discussion}

During this six-year period, over 4500 patients were successfully treated using our combination therapy. Overall, there were no severe complications, with an overall minor complication rate of $4.9 \%$, characterized by slight pain and bleeding due to injection, slight palpitation, swelling, hair growth in undesirable areas such as face and extremities, headache, dizziness, and itching.

A combination of these therapeutic options offers safe and highly effective treatment for FPHL with minimal complications, and is recommended as a useful treatment for FPHL. Our highly efficacious combination therapy, consisting of a daily oral spironolactone, oral and topical minoxidil, and a monthly injectable treatment is well accepted with minimal complications. 
Spironolactone is used to treat FPHL and hirsutism. It acts as an androgen antagonist by blocking androgen receptors, as well as inhibiting ovarian androgen production [8]. Postural hypotension, electrolyte disturbances, menstrualir regularities, fatigue, urticaria, breast tenderness, and hematological disturbances are widely known as the side effect of spironolactone. The usual daily dose of spironolactone is 100 - $200 \mathrm{mg}$. A recent open intervention study concluded that spironolactone $200 \mathrm{mg} /$ day was equally effective in either restoring hair growth or preventing further progression of hair loss compared with cyproterone acetate at a dose of either $50 \mathrm{mg} /$ day or $100 \mathrm{mg} /$ day given for 10 days every menstrual cycle [9]. In this study we have prescribed $25 \mathrm{mg}$ oral spironolactone once daily, which is quite a low dose compared to other studies, appeared to be sufficient to induce the satisfactory effect of hair regrowth in Japanese female with minimal complications.

Minoxidil for FPHL has been proven to be effective in many studies [5] [6] [10] [11] [12]. Minoxidil induces cutaneous blood flow due to vasodilatory properties [13], and up-regulates vascular endothelial growth factor (VEGF), which helps in maintaining dermal papilla vasculature and hair growth [14]. Minoxidil also promotes hair regrowth through its action to open potassium channels [15]. The effect on the cell cycle is to initiate the onset of anagen (and thereby shorten telogen duration) and to prolong the duration of anagen by delaying initiation of catagen [5]. Minoxidil also enhances hair growth by increasing the production of prostaglandin E2 through stimulation of prostaglandin endoperoxide synthase-1 [16]. Several studies have shown the effect of topical minoxidil in promoting hair growth [17] [18] [19]. A five-year follow up with topical minoxidil has shown the sustained effect of minoxidil with long term use [20].

Both spironolactone and minoxidil arrest progression of hair loss and stimulate partial regrowth of hair. They are effective but require continuous use to maintain the effect.

There are some patients who were not satisfied with the results because they could not continue the treatment financially even though varying degrees of improvements were observed in every patient. Combining medications with different mechanisms of action enhances the efficacy of FPHL treatments. Topical antiandrogens, prostaglandin analogues, topical antifungals, growth factors, laser treatment, and hair transplantation would be hopeful, additional and medical treatments for FPHL. Further studies are necessary to determine if a higher dose or increased frequency of injectable treatments may enhance the effects, and whether additional treatments or other treatments could provide results superior than our combination therapy alone.

\section{Limitations}

Lack of a control group and lack of a comparison between dosage strengths and frequencies limit the significance of our findings. 


\section{Conclusion}

FPHL is common among women as they age, and the condition is progressive without treatment. Hair loss in women has a various psychosocial impact on the affected individual and effective treatments have been required. Our combination therapy offers safe and highly efficacious treatment for FPHL with minimal complications, and is recommended as a significant treatment for FPHL.

\section{Conflicts of Interest}

The authors declare no conflicts of interest regarding the publication of this paper.

\section{References}

[1] Dinh, Q.Q. and Sinclair, R. (2007) Female Pattern Hair Loss: Current Treatment Concepts. Clinical Interventions in Aging, 2, 189-199. https://www.dovepress.com/articles.php?article id=162

[2] Gan, D.C. and Sinclair, R. (2005) Prevalence of Male and Female Pattern Hair Loss in Maryborough. Journal of Investigative Dermatology Symposium Proceedings, 10, 184-189. https://doi.org/10.1111/j.1087-0024.2005.10102.x

[3] Cash, T.F., Price, V.H. and Savin, R.C. (1993) Psychological Effects of Androgenetic Alopecia on Women: Comparisons with Balding Men and with Female Control Subjects. Journal of the American Academy of Dermatology, 29, 568-575. https://doi.org/10.1016/0190-9622(93)70223-G

[4] Van Neste, D.J. and Rushton, D.H. (1997) Hair Problems in Women. Clinics in Dermatology, 15, 113-125. https://doi.org/10.1016/S0738-081X(96)00114-9

[5] Cranwell, W. and Sinclair, R. (2000) Male Androgenetic Alopecia. MDText.com, Inc., South Dartmouth.

[6] Choe, S.J., Lee, S., Choi, J. and Lee, W.S. (2017) Therapeutic Efficacy of a Combination Therapy of Topical $17 \alpha$-Estradiol and Topical Minoxidil on Female Pattern Hair Loss: A Noncomparative, Retrospective Evaluation. Annals of Dermatology, 29, 276-282. https://doi.org/10.5021/ad.2017.29.3.276

[7] Tanaka, Y., Aso, T., Ono, J., Hosoi, R. and Kaneko, T. (2018) Androgenetic Alopecia Treatment in Asians. The Journal of Clinical and Aesthetic Dermatology, 11, 32-35. https://www.ncbi.nlm.nih.gov/pmc/articles/PMC6057731

[8] Shaw, J.C. (1996) Antiandrogen Therapy in Dermatology. International Journal of Dermatology, 35, 770-776. https://doi.org/10.1111/j.1365-4362.1996.tb02970.x

[9] Sinclair, R., Wewerinke, M. and Jolley, D. (2005) Treatment of Female Pattern Hair Loss with Oral Antiandrogens. British Journal of Dermatology, 152, 466-473. https://doi.org/10.1111/j.1365-2133.2005.06218.x

[10] Blume-Peytavi, U., Hillmann, K., Dietz, E., Canfield, D. and Garcia Bartels, N. (2011) A Randomized, Single-Blind Trial of 5\% Minoxidil Foam Once Daily versus 2\% Minoxidil Solution Twice Daily in the Treatment of Androgenetic Alopecia in Women. Journal of the American Academy of Dermatology, 65, 1126-1134e2. https://doi.org/10.1016/j.jaad.2010.09.724

[11] van Zuuren, E.J., Fedorowicz, Z. and Carter, B. (2012) Evidence-Based Treatments for Female Pattern Hair Loss: A Summary of a Cochrane Systematic Review. British Journal of Dermatology, 167, 995-1010. 
https://doi.org/10.1111/j.1365-2133.2012.11166.x

[12] Gupta, A.K. and Charrette, A. (2015) Topical Minoxidil: Systematic Review and Meta-Analysis of Its Efficacy in Androgenetic Alopecia. SKINmed Journal, 13, 185-189.

https://www.ncbi.nlm.nih.gov/pubmed/?term=12.\%09Gupta+AK\%2C+Charrette+ A.+Topical+minoxidil\%3A+systematic +review

[13] Wester, R.C., Maibach, H.I., Guy, R.H. and Novak, E. (1984) Minoxidil Stimulates Cutaneous Blood Flow in Human Balding Scalps: Pharmacodynamics Measured by Laser Doppler Velocimetry and Photopulse Plethysmography. The Journal of Investigative Dermatology, 82, 515-517. https://doi.org/10.1111/1523-1747.ep12261084

[14] Lachgar, S., Charveron, M., Gall, Y. and Bonafe, J.L. (1998) Minoxidil Upregulates the Expression of Vascular Endothelial Growth Factor in Human Hair Dermal Papilla Cells. The British Journal of Dermatology, 138, 407-411. https://doi.org/10.1046/j.1365-2133.1998.02115.x

[15] Buhl, A.E., Conrad, S.J., Waldon, D.J. and Brunden, M.N. (1993) Potassium Channel Conductance as a Control Mechanism in Hair Follicles. The Journal of Investigative Dermatology, 101, 148S-152S. https://doi.org/10.1111/1523-1747.ep12363290

[16] Colombe, L., Vindrios, A., Michelet, J.F. and Bernard, B.A. (2007) Prostaglandin Metabolism in Human Hair Follicle. Experimental Dermatology, 16, 762-769. https://doi.org/10.1111/j.1600-0625.2007.00586.x

[17] Katz, H.I., Hien, N.T., Prawer, S.E. and Goldman, S.J. (1987) Long-Term Efficacy of Topical Minoxidil in Male Pattern Baldness. Journal of the American Academy of Dermatology, 16, 711-718. https://doi.org/10.1016/S0190-9622(87)70092-9

[18] Rietschel, R.L. and Duncan, S.H. (1987) Safety and Efficacy of Topical Minoxidil in the Management of Androgenetic Alopecia. Journal of the American Academy of Dermatology, 16, 677-685. https://doi.org/10.1016/S0190-9622(87)70087-5

[19] Price, V.H., Menefee, E. and Strauss, P.C. (1999) Changes in Hair Weight and Hair Count in Men with Androgenetic Alopecia, after Application of 5\% and 2\% Topical Minoxidil, Placebo, or No Treatment. Journal of the American Academy of Dermatology, 41, 717-721. https://doi.org/10.1016/S0190-9622(99)70006-X

[20] Olsen, E.A., Weiner, M.S., Amara, I.A. and DeLong, E.R. (1990) Five-Year Follow-Up of Men with Androgenetic Alopecia Treated with Topical Minoxidil. Journal of the American Academy of Dermatology, 22, 643-646. https://doi.org/10.1016/0190-9622(90)70089-Z 